\title{
Virtual Reality System for Multiple Sclerosis Rehabilitation using KINECT
}

\author{
J A Lozano-Quilis ${ }^{1}$, H Gil-Gómez ${ }^{1}$, J A Gil-Gómez ${ }^{1}$ \\ ${ }^{1}$ Instituto de Automática e Informática Industrial \\ Universitat Politècnica de València \\ C. Vera S/N, 46022, Valencia, SPAIN
}

\author{
S Albiol-Pérez ${ }^{2}$, G Palacios ${ }^{2}$, Habib M. Fardoum² ${ }^{3}$, \\ Abdulfattah S. Mashat ${ }^{3}$ \\ ${ }^{2}$ Dpto. de Informática e Ingeniería de Sistemas \\ Universidad de Zaragoza \\ C. Ciudad Escolar S/N, 44003, Teruel, SPAIN \\ ${ }^{3}$ Information Systems Department King Abdulaziz \\ University (KAU) Jeddah, Saudi Arabia
}

\begin{abstract}
REOVIEM is a system that use the potencial of virtual reality (VR) and natural user interfaces (NUI) to offer to patients with multiple sclerosis (MS) an intuitive and motivating way to perform several motor rehabilitation exercise. Moreover, REMOVIEM offer to the therapists a new motor rehabilitation tool that it allows control the rehabilitation process, and evaluate the progress of the patients. On the other hand, REMOVIEM is a low cost system, a feature that it facilitates its integration in the clinical rehabilitation centers.
\end{abstract}

Keywords; Multiple Sclerosis; Motor Rehabilitation; Virtual Reality; Natural Interfaces.

\section{INTRODUCTION}

The methods used for motor rehabilitation of patients with neurological problems requires the execution of several rehabilitation exercises. Basically, these methods have two fundamental problems. First, they propose to exercise motor skills performing the exercises in an insistent and repetitive way, that it is a very little motivating way and it decreases the interest of the patient to perform them. Secondly, they require to exercise motor skills in specific centers with qualified personnel to supervise at all times, its correct performance.

The use of new technologies, like VR and NUI, on the motor rehabilitation of patients with neurological disorders, are well documented [1]. The advantages of VR compared to the traditional methods used for this purpose, are basically three. First, its capacity to recreate several rehabilitation exercises to be performed by the patient, in a virtual way (virtual rehabilitation exercises). Secondly, its capacity to configure the features of the rehabilitation exercises, to control their performance, and also to obtain relevant data from patient to perform them. Finally, its capacity to facilitate the interaction between patient and system by means of a wide variety of devices. On the other hand, several studies have demonstrated that offering the virtual rehabilitation exercises in a similar way to the games, it si possible to obtain a greater efficiency of the rehabilitation process motivating the patients to perform the rehabilitation exercises, and also increasing their adherence to the treatment [2], [3], [4]. The advantages of using NUI for game consoles with virtual rehabilitation systems also they are well documented [5], [6], [7], [8], [9], [10].

It is true there are some studies focused in the use of technical solutions for the rehabilitation of patients with MS. For instance, the use of VR and Augmented Reality (AR) for the rehabilitation of the gait [11], or the use of NUI for the rehabilitation of patients with reduced motor skills [12]. But, in our opinion, this approach of using VR and NUI for the motor rehabilitation of patients with MS has not yet been widely used, and we think it can offer many possibilities.

In the case of patients with MS, the performance of motor rehabilitation exercises in the rehabilitation process it is even more important, especially if the disease is diagnosed early enough. These patients still have motor skills, and we think they could begin a rehabilitation process, performing virtual rehabilitation exercises, in a similar way to the games, and interacting with them in an easy and intuitive way. That is, using natural user interfaces similar to used in games consoles. REMOVIEM, the system proposed in this paper, is just an attempt to include all these features in one motor rehabilitation

\section{METHODS}

REMOVIEM has two parts: software and hardware. The program consists of several virtual environments (VEs) designed and developed to allow the patient to perform several motor rehabilitation exercises. In the other hand, with the software the therapist can select and configure the exercises to be performed by the patient, and even view and evaluate the results. The hardware is composed of several low cost devices used by visualize VEs in a immersiver way, and also to interact with them in a very intuitve way. This "low cost" component of the devices allows its integration in the clinical rehabilitation centers in a easy way.

\section{A. Software: Virtual Environments}

REMOVIEM has three motor rehabilitation exercises, that is, three VEs: TouchBall, TakeBall, and StepBall 


\section{TouchBall:}

Objective: to work on the balance and weight transfer of the patient, to perform lateral movements of the trunk. Both issues are essential in order to achieve an improvement in gait and, in this way, to perform a right walk.

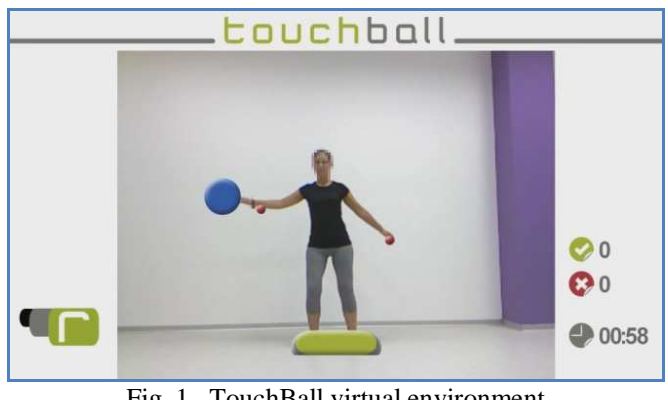

How the VE works: In the Figure 1 it is possible to see how this VE works. The patient, in a standing or sitting way, see at either sides, and different heights, several virtual objects that they must be touched with his hands, before they disappear, and not moving the foots outside of a predefined zone. In order to make this, the patient has a long time previously established by the therapist. On the other hand, the VE counts the successes and mistakes of the patient. If the patient touch the virtual object before it disappears, with the hand indicated by the system, and not moving their feet outside of the predefined limiting zone, he obtains a sucess. But, if the patient does not touch the virtual object before it disappears, he does not touch it with the hand indicated by the system, or he moves their feet outside of the predefined limiting zone, he obtains a mistake.

\section{TakeBall:}

Objective: to work in the patient the Diagonals of Kabat $M M S S$, very important in neurological rehabilitation, because they work in complete movements of the upper limbs, requiring only good coordination for its implementation.

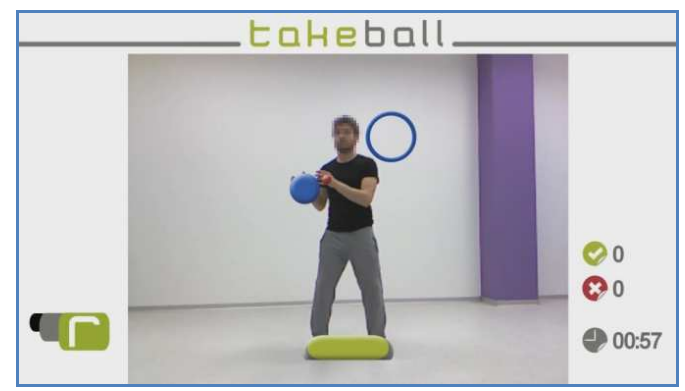

Fig. 2. TakeBall virtual environment

How the VE works: In the Figure 2 it is possible to see how this VE works. The patient, in a standing or sitting way, see several virtual objects that they must be moved with both hands, from a initial position in which they appear, until the final position indicated by the system, following a path also indicated by the system. In order to make this, the patient has a long time previously established by the therapist. On the other hand, the VE counts the successes and mistakes of the patient. If the patient moves the objet from the initial position until the final position, before it disappears, and following the path established by the system, he obtains a sucess. But, if the patient does not move the object before it disappears, or he does not move it following the path established by the system, he obtains a mistake.

\section{StepBall:}

Objective: to work in the patient the balance and weight transfer, in order to perform lateral movements with monopodal load.

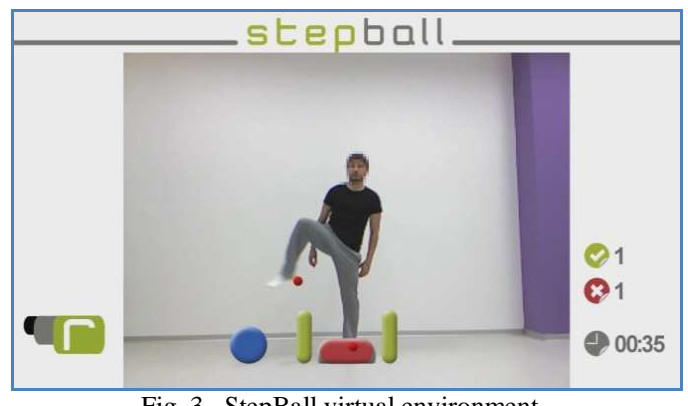

Fig. 3. StepBall virtual environment

How the VE works: In the Figure 3 it is possible to see how this VE works. The patient, in a standing way, see at either sides, and to the ground level, several virtual objects that they must be stepped with his foots, before such objects disappear. In order to step them, the patient performs a lateral movement of the leg closest to the virtual object, lifting it just enough to avoid throwing another virtual object, as an obstacle, stand between the patient's leg and the virtual object to step. In orther to mahe this, the patient has a long time previously established by the therapist. On the other hand, the VE counts the successes and mistakes of the patient. If the patient step the virtual object before it disappears, and not throwing the virtual obstacle to avoid, he obtains a sucess. But, if the patient threw the virtual obstacle to avoid, or (even avoiding it) does not step the virtual object before it disappears, he obtains a mistake.

All VEs follow the same operating system. Initially there is a window with the VE settings menu. In the Figure 4 it is possible to see this menu allows to the therapist to modify the parameters of the motor rehabilitation exercise considering the feautres of the patient. In the Figure 5 we see after configuration, the patient see a sequence of images explanatory about the interaction with the VE. The goal of these images is the patient, in a simple and intuitive, understand as he/she must interact with the system in order to perform the VE. Afther there is a window, showed in the Figure 6, in which the patient can see how the system detects the skeleton of the patient body, 
and as it also detects he/she is correctly placed to carry out the exercise. In the Figure 7 it is possible to see when the system detects that the patient is properly positioned, it start a countdown that it will allow the patient to prepare for the start of the execution of the exercise. Finally, in the Figure 8 we see after the execution of the exercise, the system shows to the patient a window with a summary of the results obtained.

\section{B. Hardware}

REMOVIEM propose a LCD/LED TV (42"-47") to watch and hear the visual and auditory contents of the virtual environments. Specifically, the patient watch himself, included in one of the virtual environments mentioned previously. In order to interact with the virtual environments, the patient use Microsoft Kinect ${ }^{\circledR}$ that it has several sensors (image, audio and depth) used to detect movements, to identify faces, or to recognize speech. REMOVIEM only use the detection all movements of the patients. For this, the patient is placed in front of the Microsoft Kinect ${ }^{\circledR}$, as a suitable distance to be able to capture completely all his body movements.

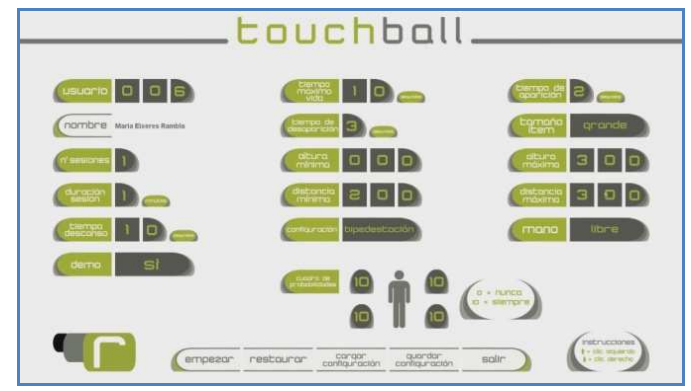

Fig. 4. Configuration virtual environments

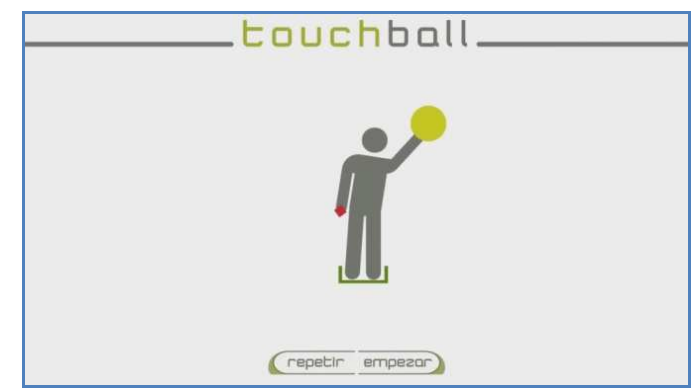

Fig. 5. Images explanatory about the interaction with the virtual environment.

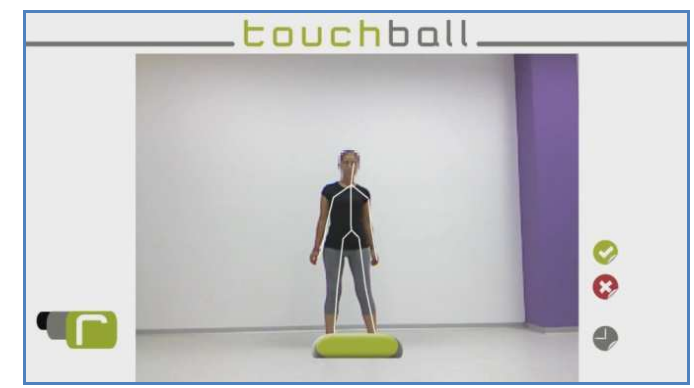

Fig. 6. The detection of the skeleton of the patient in the virtual environment.

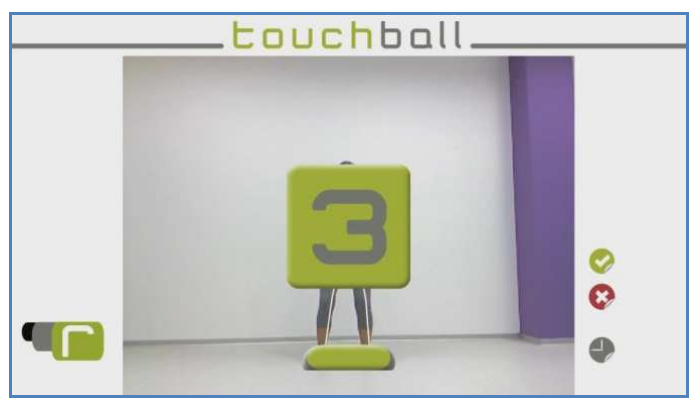

Fig. 7. The countdown before the execution of the virtual environment

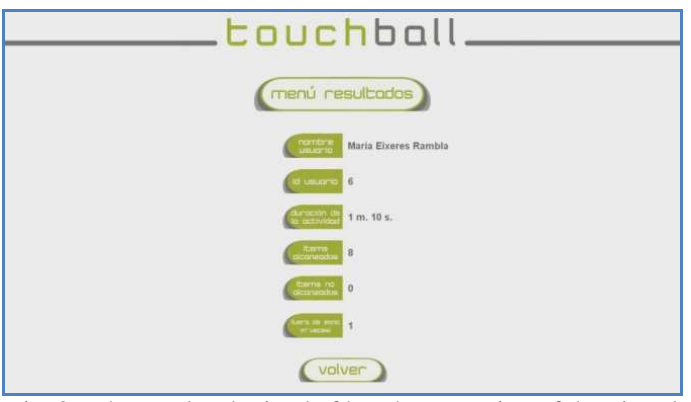

Fig. 8. The results obtained afther the execution of the virtual environment.

\section{CONCLUSIONS}

REMOVIEM is in a clinical evaluation phase. This phase is being carried out by the professionals of the rehabilitation of patients with MS of the Association of Multiple Sclerosis of Castellon (Spain), that also they have participated in the specifications of the feautres of the system and the virtual evironments. In this way, it will be possible to know the clinical utility of the system, and the acceptance degree of patients.

In order to carry out this clinical evaluation, we have selected 12 patients, following these criteria. Inclusion Criteria: a) men and women between 25 and 35 years old, $b$ ) with relapsing-remitting and secondary progressive multiple sclerosis, c) a minimum score of 6 on all items of the domain of the Functional Independence Measure (FIM), d) patients dont need assistive devices for ambulation or at most a cane of a point, e) and they dont have cognitive impairments. Exclusion Criteria: a) patients who are in bud stage, $b$ ) or they require assistive devices for ambulation. The patients selected have been divided into two groups (control group and experimental group), everyone of 6 patients. Every patient will perform 10 hour sessions (one per week). In order to assess the static and dynamic balance abilities of the patients we will use the Tinetti Test (TT) and 10-meter walk test (10 MWT). Moreover, also we will use a feedback questionnaire (SFQ) in order to know the opinion of the patient in relation with the treatment. 


\section{ACKNOWLEDGMENT}

The authors wish to thank the patients and professional of the rehabilitation process of the Association of Multiple Sclerosis of Castellon (Spain) for their collaboration. This contribution was funded partially by Generalitat Valenciana ("Ajudes per a la realitzación de projectes d'I+D per a grups d'investigación emergents", projecte GV/2012/069).

\section{REFERENCES}

[1] S. A. Adamovich, G. G. Fluet, E. Tunik and A. S. Merians, "Sensoriomotor training in virtual reality: a review", Neurorehabilitation, 25(1), pp. 29-44., 2009.

[2] B. S. Lange, S. M. Flynn, R. Proffit, C. Y. Chang, A. A. Rizzo, "Development of an interactive game-based rehabilitation tool for dynamic balance training", Topics in Stroke rehabilitation, 17 (5), pp. 345-352, 2010.

[3] S. M. Flynn and B. S. Lange, "Games for the rehabilitation: the voice of the players", Intl. Conf. Disability, Virtual Reality \& Associated Technologies (ICDVRAT 2010), pp. 185-194, 2010.

[4] J. A. Lozano-Quilis, J. Montesa, M. C. Juan, M. ALcañiz, B. Rey, J. A. Gil-Gómez, J. M. Martínez, A. Gaggioli, F. Morganti, "VR-Mirror: a virtual reality system for mental practice in post-stroke rehabilitation", Smart Graphics 2005, pp. 241-245, 2005.

[5] B. S. Lange, P. Requejo, S. M. Flynn, A. A. Rizzo, F. J. Valero-Cuevas, L. Baker and C. Winstein, "The potential of virtual reality and gaming to assist successful aging with disability", Physical Medicine Rehabilitation Clinics of North America, 21(2), pp. 339-356, 2010.

[6] Y. P. Wuang, C. S. Chiang, C. Y. Su and C. C. Wang, "Effectiveness of virtual reality using Wii gaming technology in children with Down syndrome", Research in Developmental Disabilities, 32 (1), pp. 312-321, 2011

[7] F. Anderson, M. Annet and W. F. Bischof, "Lean on Wii: physical rehabilitation with virtual reality Wii peripherals", Studies in Health Technology and Informatics, 154, pp. 229-234, 2010.

[8] J. A. Gil-Gómez et al., "Effectiveness of a Wii balance board-based system (eBaViR) for balance rehabilitation: A pilot randomized clinical trial in patients with acquired brain injury", Journal of Neuroengineering and Rehabilitation 8: 30. 2011.

[9] J. A. Gil-Gómez, M. Alcañiz, J. Montesa, M. Ferrer, J. Chirivella, E. Noé, C. Colomer, "Low-cost virtual motor rehabilitation system for standing exercises", Virtual Rehabilitation 2007, pp. 34-38, 2007.

[10] Yao-Jen Chang, Shu-Fang Chen, Jun-Da Huang, "A kinect-based system for phisical rehabilitation: a pilot study for young adults with motor disabilities", Research in Developmental Disabilities, 32(6), pp. 25672570, 2011.

[11] A. Da Gama, T. Chavez, L. Figueiredo, V. Teichrieb, "Poster: improving motor rehabilitation process through a natural interaction based system using kinect sensor", IEEE Symposium on 3D User Interfaces 2012, pp. $145-146,2012$

[12] Y. Baram and A. Miller, "Virtual reality cues for improvement of gait in patients with multiple sclerosis", Neurology, 66 (2), pp. 178-181, 2006.

[13] G. D. Fulk, "Locomotor training and virtual reality-based balance training for an individual with multiple sclerosis: a case report", Journal of Neurologic Physical Therapy, 29 (1), pp. 34-42, 2005. 